\title{
Gastric cancer mortality rates among US and foreign-born persons: United States 2005-2014
}

\author{
Benjamin D. Hallowell ${ }^{1}$ Meheret Endeshaw ${ }^{1} \cdot$ Virginia Senkomago $^{1} \cdot$ Hilda Razzaghi $^{1} \cdot$ Matthew T. McKenna $^{2}$. \\ Mona Saraiya ${ }^{1}(1)$
}

Received: 26 September 2018 / Accepted: 24 February 2019 / Published online: 4 March 2019

(c) The International Gastric Cancer Association and The Japanese Gastric Cancer Association 2019

\begin{abstract}
Background Historically, foreign-born individuals in the US have had an elevated risk of dying from gastric cancer when compared to US-born individuals. This is primarily due to factors that occur prior to their immigration to the US, including diet and underlying risk of $\mathrm{H}$. pylori infection.

Methods National mortality data from 2005 to 2014 were obtained from the CDC's National Center for Health Statistics. Annual population estimates were obtained from the US Census Bureau's American Community Survey for foreign-born and US-born persons. Age-adjusted gastric cancer mortality rates and rate ratios (RR) were calculated stratified by birth place, age, race/ethnicity, and geographic location.

Results From 2005 to 2014, 111,718 deaths from malignant gastric cancer occurred in the US, of which 24,583 (22\%) occurred among foreign-born individuals. Overall, foreign-born individuals had higher mortality rates compared with USborn individuals (RR 1.82; 95\% CI 1.80,1.85) and this difference remained after stratifying by sex, age, and geographic location. However, this finding was primarily driven by the low rate of gastric cancer mortality among US-born whites, with similar mortality rates observed among all other foreign-born and US-born groups. Gastric cancer mortality rates significantly decreased during the study period overall (AAPC $-2.50 ; 95 \% \mathrm{CI}-3.21,-1.79$ ) with significant declines observed among US-born (AAPC - 2.81; 95\% CI - 3.55, -2.07) and the foreign-born (AAPC - 2.53; 95\% CI - 3.20, - 1.86) population.

Conclusions Efforts directed at reducing the prevalence of gastric cancer risk factors could help reduce the elevated burden observed among foreign-born individuals and US-born minority groups.
\end{abstract}

Keywords Gastric cancer $\cdot$ Mortality $\cdot$ Immigrants $\cdot$ United States

\section{Introduction}

Gastric cancer is the second leading cause of cancer-related deaths worldwide, with 984,00 new cases and 841,000 deaths occurring annually [1]. While dietary factors, genetic susceptibility, and smoking can increase gastric cancer risk,

The findings and conclusions in this report are those of the authors and do not necessarily represent the official position of the Centers for Disease Control and Prevention.

Mona Saraiya

yzs2@cdc.gov

1 Division of Cancer Prevention and Control, Centers for Disease Control and Prevention, 4770 Buford Highway, Atlanta, GA 30341, USA

2 Emory University School of Medicine, Atlanta, GA, USA most cases $(89 \%)$ are attributed to H. pylori infection [2-4]. Worldwide, over half of the world's population (4.4 billion) is infected with $H$. pylori, with the prevalence varying dramatically by geographic region [Africa $(79.1 \%)$, Latin America (63.4\%), Asia (54.7\%), Europe (34.3-62.8\%), and North America (37.1\%)] [5]. In contrast to this distribution, almost $75 \%$ of gastric cancer cases occur in Asia, with over half of all gastric cancer patients coming from China, Japan, and Korea [1,5].

In the United States (US), gastric cancer is less frequent, with 23,695 new cases and 11,330 deaths occurring in 2015 [6]. Due to factors that occur prior to their immigration to the US (diet and underlying risk of $H$. pylori infection), foreign-born individuals in the US have an elevated risk of developing and dying from gastric cancer when compared to US-born individuals [7-9]. To address this issue, some groups are beginning to recommend $H$. pylori screening for 
first-generation immigrants who migrate from high burden countries [10]. To better address this health disparity, we utilized recent cancer mortality data to describe gastric cancer mortality among the foreign-born population.

\section{Methods}

Population-based mortality data from 2005 to 2014 were obtained from the National Center for Health Statistics (NCHS) for the 50 states and the District of Columbia. NCHS collects information on sex, race, ethnicity, place of birth, age at death, state of residence, and cause of death from death certificates filed in each state. Individuals who were born in one of the 50 states, the District of Columbia, or the US territories were categorized as US-born. All other cases with recorded places of birth were categorized as foreign-born [11]. Deaths for malignant gastric cancer were selected using the International Classification of Disease version 10 (ICD-10) code $\mathrm{C} 16$ based on underlying cause of death. Cases with no recorded place of birth $(1.11 \%)$ were excluded from all analyses.

Annual population estimates were obtained from the US Census Bureau's American Community Survey (ACS). Annual ACS public-use microdata sample files were used to estimate the US-born and foreign-born population by age, race/ethnicity, sex, and census region using provided person weights.

Mortality data and population estimates from 2005 to 2014 were formatted using the Surveillance Epidemiology End Results Program SEER*Prep [12] software to create a SEER*Stat database [13]. We calculated age-adjusted mortality rates adjusting to the 2000 US standard population. SEER*Stat was used to calculate mortality rate ratios (RR) by nativity status, age, race/ethnicity, and geographic location. Joinpoint was used to calculate the average annual percent change (AAPC) from 2005 to 2014.

\section{Results}

From 2005 to 2014, 111,718 deaths from malignant gastric cancer occurred in the US, of which 24,583 (22\%) occurred among the foreign-born population. Overall, foreign-born individuals had higher mortality rates when compared with US-born individuals (RR 1.82; 95\% CI 1.80, 1.85) and the differences remained after stratifying by sex, age, and geographic location (Table 1).

Foreign-born males who were non-Hispanic white (RR $2.01 ; 95 \%$ CI $1.95,2.08$ ) had higher mortality rates when compared with US-born non-Hispanic whites. In contrast, foreign-born men who were non-Hispanic black (RR 0.87; 95\% CI 0.80, 0.94), non-Hispanic Asian/Pacific Islander (RR
0.98; 95\% CI 0.90, 1.07), and Hispanic (RR 0.99; 95\% CI $0.94,1.04)$ had a similar or lower mortality rates when compared to their US-born counterparts. Non-Hispanic white (RR 1.91; 95\% CI 1.84, 1.99), non-Hispanic Asian/Pacific Islander (RR 1.25; 95\% CI 1.13, 1.40) and Hispanic (RR $1.10 ; 95 \%$ CI 1.04, 1.16) foreign-born women had higher mortality rates compared to their US-counterparts, with no excess risk observed among foreign-born non-Hispanic blacks. When examined by race/ethnicity overall, the lowest mortality rates were observed among US-born non-Hispanic whites, with similar rates observed among all other US and foreign-born race/ethnicity groups (Fig. 1).

Gastric cancer mortality rates significantly decreased during the study period overall (AAPC $-2.50 ; 95 \% \mathrm{CI}-3.21$, - 1.79) with significant declines observed among US-born (AAPC $-2.81 ; 95 \% \mathrm{CI}-3.55,-2.07$ ) and the foreign-born (AAPC $-2.53 ; 95 \%$ CI $-3.20,-1.86$ ) population. With the exception of foreign-born Hispanics, cancer mortality rates significantly decreased among all racial/ethnic groups (Fig. 1).

\section{Discussion}

Foreign-born individuals in the US had a higher rate of gastric cancer mortality when compared to US-born individuals; however, this was primarily driven by the low rate of gastric cancer mortality observed among US-born whites. $H$. pylori is considered a necessary, but not sufficient cause for gastric cancer, and the most recent reviews of studies using sensitive assays for this bacterium conclude that at least $89 \%$ of such cancers are attributable to $H$. pylori infection [4]. Therefore, the elevated mortality rates observed among foreign-born individuals and US-born minority groups are likely reflective of elevated $H$. pylori infection rates in these populations, which have been previously documented $[5$, 14].

To reduce the burden of gastric cancer among foreign-born and US-born minority groups, efforts could be directed at reducing the prevalence of $H$. pylori, diet modification, and improvements in socioeconomic position and hygiene levels [3]. Recently published consensus guidelines recommended that "individuals who are firstgeneration immigrants from high prevalence areas be tested for H. pylori" [10]. The authors rated this as a strong recommendation based on moderate quality evidence. The authors also "suggested" that Latino and African American racial or ethnic groups "may be" considered for $H$. pylori testing (a weak recommendation based on low quality evidence) due to their high risk of infection [10]. The authors strongly emphasized that testing methods which identify active infection, such as stool antigen testing and the urea breath testing, should be used and that serological 
Table 1 Gastric cancer mortality rates in the US by demographic characteristics: 2005-2014. Source: National Vital Statistics System, Mortality Files

\begin{tabular}{|c|c|c|c|c|c|}
\hline & \multicolumn{2}{|l|}{ US-born } & \multicolumn{2}{|l|}{ Foreign-born } & \multirow{2}{*}{$\begin{array}{l}\text { Rate ratios }^{c} \\
\text { Rate ratio }(95 \% \mathrm{CI})\end{array}$} \\
\hline & No. of cases & Average annual rate $(95 \% \mathrm{CI})^{\mathrm{a}}$ & No. of cases & Average annual rate $(95 \% \mathrm{CI})^{\mathrm{a}}$ & \\
\hline Overall $^{\mathrm{b}}$ & 87,135 & $3.05(3.03,3.07)$ & 24,583 & $5.56(5.49,5.63)$ & $1.82 *(1.80,1.85)$ \\
\hline \multicolumn{6}{|l|}{ Sex } \\
\hline Male & 52,527 & $4.23(4.20,4.27)$ & 13,691 & $7.39(7.26,7.52)$ & $1.75 *(1.71,1.78)$ \\
\hline Female & 34,608 & $2.13(2.11,2.15)$ & 10,892 & $4.30(4.22,4.38)$ & $2.02 *(1.98,2.06)$ \\
\hline \multicolumn{6}{|l|}{ Age (years)—male } \\
\hline$<35$ & 494 & $0.08(0.08,0.09)$ & 260 & $0.18(0.16,0.21)$ & $2.10 *(1.80,2.53)$ \\
\hline $35-49$ & 3510 & $1.32(1.28,1.37)$ & 1496 & $2.28(2.17,2.40)$ & $1.73 *(1.62,1.83)$ \\
\hline $50-64$ & 13,819 & $5.66(5.56,5.75)$ & 3704 & $9.30(9.00,9.60)$ & $1.64 *(1.58,1.70)$ \\
\hline $65-79$ & 20,673 & $18.41(18.16,18.67)$ & 4964 & $31.62(30.74,32.53)$ & $1.72 *(1.66,1.77)$ \\
\hline $80+$ & 14,031 & $39.57(38.92,40.23)$ & 3267 & $72.81(70.33,75.35)$ & $1.84 *(1.77,1.91)$ \\
\hline \multicolumn{6}{|l|}{ Age (years)—female } \\
\hline$<35$ & 414 & $0.07(0.06,0.08)$ & 297 & $0.22(0.20,0.26)$ & $3.14 *(2.69,3.76)$ \\
\hline $35-49$ & 2256 & $0.84(0.80,0.87)$ & 1462 & $2.25(2.14,2.37)$ & $2.68 *(2.51,2.87)$ \\
\hline $50-64$ & 6530 & $2.53(2.47,2.59)$ & 2470 & $5.65(5.43,5.88)$ & $2.24 *(2.13,2.34)$ \\
\hline $65-79$ & 11,401 & $8.52(8.36,8.67)$ & 3452 & $16.27(15.73,16.82)$ & $1.91 *(1.84,1.99)$ \\
\hline $80+$ & 14,007 & $21.80(21.44,22.17)$ & 3211 & $39.07(37.73,40.45)$ & $1.79 *(1.72,1.86)$ \\
\hline \multicolumn{6}{|l|}{ Race/ethnicity_male } \\
\hline Non-Hispanic White & 37,210 & $3.52(3.49,3.56)$ & 4506 & $7.10(6.89,7.31)$ & $2.01 *(1.95,2.08)$ \\
\hline Non-Hispanic Black & 10,431 & $9.72(9.52,9.92)$ & 878 & $8.45(7.82,9.12)$ & $0.87 *(0.80,0.94)$ \\
\hline Non-Hispanic API & 717 & $8.33(7.72,8.97)$ & 3514 & $8.18(7.89,8.48)$ & $0.98(0.90,1.07)$ \\
\hline Hispanic & 3542 & $7.23(6.98,7.49)$ & 4772 & $7.14(6.91,7.37)$ & $0.99(0.94,1.04)$ \\
\hline \multicolumn{6}{|l|}{ Race/ethnicity_female } \\
\hline Non-Hispanic White & 24,042 & $1.74(1.72,1.76)$ & 3516 & $3.89(3.75,4.03)$ & $2.23 *(2.15,2.32)$ \\
\hline Non-Hispanic Black & 7188 & $4.49(4.39,4.60)$ & 684 & $4.55(4.20,4.93)$ & $1.01(0.93,1.10)$ \\
\hline Non-Hispanic API & 459 & $3.98(3.61,4.38)$ & 2938 & $5.00(4.81,5.19)$ & $1.25^{*}(1.13,1.40)$ \\
\hline Hispanic & 2539 & $3.94(3.79,4.10)$ & 3737 & $4.33(4.19,4.48)$ & $1.10^{*}(1.04,1.16)$ \\
\hline \multicolumn{6}{|l|}{ US census region-male } \\
\hline Northeast & 9847 & $4.37(4.29,4.46)$ & 3712 & $8.36(8.08,8.64)$ & $1.91 *(1.84,1.99)$ \\
\hline Midwest & 11,896 & $3.98(3.91,4.06)$ & 1444 & $7.51(7.11,7.93)$ & $1.89 *(1.78,2.00)$ \\
\hline South & 20,703 & $4.40(4.34,4.46)$ & 3381 & $6.14(5.92,6.37)$ & $1.40 *(1.34,1.45)$ \\
\hline West & 10,081 & $4.07(3.99,4.15)$ & 5154 & $7.65(7.44,7.88)$ & $1.88 *(1.82,1.95)$ \\
\hline \multicolumn{6}{|c|}{ US census region—female } \\
\hline Northeast & 6944 & $2.18(2.12,2.23)$ & 2625 & $4.19(2.03,4.36)$ & $1.92 *(1.84,2.02)$ \\
\hline Midwest & 7715 & $1.94(1.90,1.98)$ & 1125 & $4.24(3.99,4.50)$ & $2.19 *(2.05,2.33)$ \\
\hline South & 13,619 & $2.25(2.21,2.29)$ & 2765 & $3.75(3.61,3.89)$ & $1.67 *(1.60,1.74)$ \\
\hline West & 6330 & $2.07(2.02,2.12)$ & 4377 & $4.82(4.68,4.97)$ & $2.33^{*}(2.24,2.42)$ \\
\hline
\end{tabular}

Gastric cancers were defined using ICD-10 code C16 based on underlying cause of death

API Asian and Pacific Islander

${ }^{a}$ Rates are age-adjusted to 2000 standard U.S. population and shown per 100,000

${ }^{\mathrm{b}}$ Columns may not sum to total because of missing values

${ }^{c}$ US-born were used as a reference group to generate rate ratios

testing should be discouraged. The recommendations also emphasize antibiotic treatment is needed for persons with active infection, and eradication in treated persons should be documented with follow-up testing. Increasing consumption of fruits, vegetables, and fiber while reducing the intake of foods that have been smoked, salted, or pickled can further reduce gastric cancer risk [2,3]. Finally, as $H$. pylori infection generally occurs from interfamilial transmission during childhood, working to improve the socioeconomic position and hygiene levels of impacted 


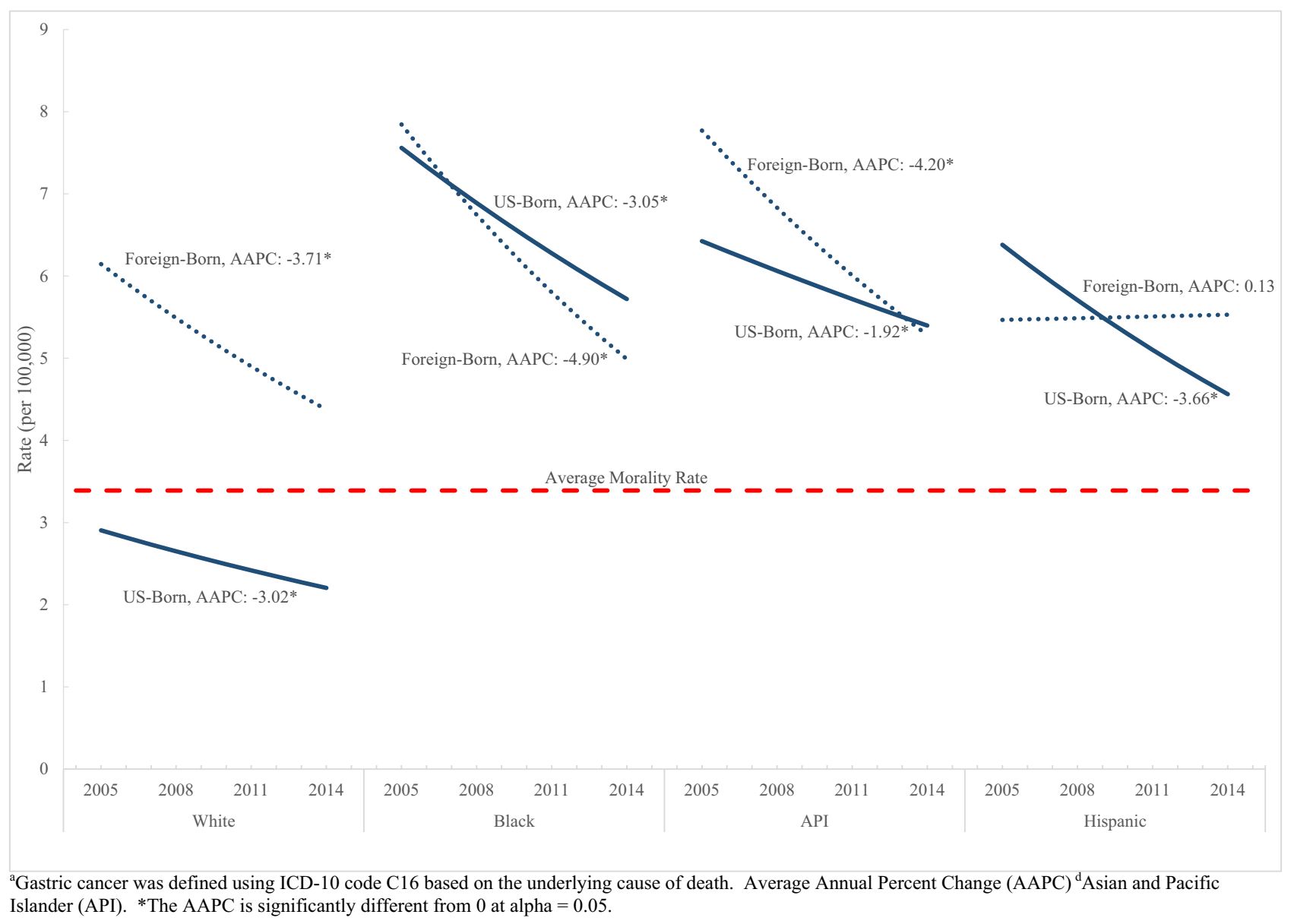

Fig. 1 Average annual percent change in gastric cancer mortality rates by birth place and race/ethnicity: United States 2005-2014. Gastric cancer was defined using ICD-10 code C16 based on the

populations can further reduce $H$. pylori's burden among affected groups $[2,3,5]$.

The significant declines in gastric cancer mortality observed among non-Hispanic whites, non-Hispanic blacks, and non-Hispanic APIs reflect global declines in gastric cancer as $H$. pylori prevalence continues to decline with improvements in socioeconomic status, hygiene levels, and healthcare access [1]. In contrast to global trends, in Mexico, the gastric cancer mortality rate has remained relatively stable since the 1990s [15]. As a majority of foreign-born Hispanics are born in Mexico, the lack of decline observed among foreign-born Hispanics likely reflects these trends.

With the identification of specific high-risk groups (e.g., first-generation immigrants from China, Japan, or Korea) directly screening for gastric cancer may be appropriate and could lower mortality risk $[4,16]$. In addition, studies conducted in high burden areas have found that $H$. pylori screening, treatment, and eradication can effectively reduce gastric cancer risk by $34 \%$ [17]. However, while gastric cancer screening programs have been successful in some Asian underlying cause of death. $A A P C$ average annual percent change, $A P I$ Asian and Pacific Islander. ${ }^{*}$ The AAPC is significantly different from 0 at alpha $=0.05$

countries, very limited data exist on their utility in lower burden countries [18]. Currently, there is no evidence from randomized trials in the United States that demonstrate a decrease in mortality from direct screening using endoscopy for gastric cancer. This paucity of evidence, and the invasive nature of endoscopic testing, has led to this service not being regularly promoted in any population in this country [4]. While many studies have described the proper screening, management, and treatment of $H$. pylori $[19,20]$, future work could evaluate the efficacy of population-based $H$. pylori screening, management, and treatment in lower burden settings and among high-risk populations [3-5].

This study's greatest strength is that it provides gastric cancer mortality rates by birth place, age, sex, race/ethnicity, and geographic region for the entire US population over the last decade. Lack of information on birthplace for foreign born was a limitation of the data used. Future work could utilize country specific information to identify countries with particular high gastric cancer mortality risk. 


\section{Compliance with ethical standards}

Conflict of interest The authors declare that they have no conflict of interest.

Research involving human participants and animals The article does not contain any studies with human or animal subjects performed by any of the authors.

\section{References}

1. Fitzmaurice $\mathrm{C}$, et al. The global burden of cancer 2013. JAMA Oncol. 2015;1(4):505-27.

2. Compare D, Rocco A, Nardone G. Risk factors in gastric cancer. Eur Rev Med Pharmacol Sci. 2010;14(4):302-8.

3. Stewart B, Wild CP. World cancer report 2014. 1. Lyon: International Agency for Research on Cancer; 2014.

4. Plummer M, et al. Global burden of cancers attributable to infections in 2012: a synthetic analysis. Lancet Glob Health. 2016;4(9):e609-16.

5. Hooi JK, et al. Global prevalence of Helicobacter pylori infection: systematic review and meta-analysis. Gastroenterology. 2017;153(2):420-9.

6. U.S. Cancer Statistics Working Group. United States Cancer statistics: 1999-2015 incidence and mortality web-based report. U.S. Department of Health and Human Services Centers for Disease Control and Prevention and National Cancer Institute, Editor; 2018.

7. Singh GK, Hiatt RA. Trends and disparities in socioeconomic and behavioural characteristics, life expectancy, and cause-specific mortality of native-born and foreign-born populations in the United States, 1979-2003. Int J Epidemiol. 2006;35(4):903-19.

8. Singh GK, Miller BA. Health, life expectancy, and mortality patterns among immigrant populations in the United States. Can J Public Health. 2004;95(3):14-21.

9. Singh GK, Siahpush M. All-cause and cause-specific mortality of immigrants and native born in the United States. Am J Public Health. 2001;91(3):392.
10. El-Serag HB, et al. Houston consensus conference on testing for Helicobacter pylori infection in the United States. Clin Gastroenterol Hepatol. 2018;16(7):992-1002.

11. Singh GK, Rodriguez-Lainz A, Kogan MD. Immigrant health inequalities in the United States: use of eight major national data systems. Sci World J. 2013;2013:512313. https://doi. org/10.1155/2013/512313.

12. Surveillance Research Program National Cancer Institute. SEER*Prep software. Bethesda: National Cancer Institute; 2016.

13. Surveillance Research Program National Cancer Institute. SEER*Stat software. Bethesda: National Cancer Institute; 2017.

14. Grad YH, Lipsitch M, Aiello AE. Secular trends in Helicobacter pylori seroprevalence in adults in the United States: evidence for sustained race/ethnic disparities. Am J Epidemiol. 2011;175(1):54-9.

15. Rizo-Ríos P, et al. Trends in cancer mortality in Mexico: 1990-2012. Revista Médica Del Hospital General De México. 2015;78(2):85-94.

16. Kim GH, et al. Screening and surveillance for gastric cancer in the United States: is it needed? Gastrointest Endosc. 2016;84(1):18-28.

17. Ford AC, et al. Helicobacter pylori eradication therapy to prevent gastric cancer in healthy asymptomatic infected individuals: systematic review and meta-analysis of randomised controlled trials. Bmj. 2014;348:g3174.

18. Leung WK, et al. Screening for gastric cancer in Asia: current evidence and practice. Lancet Oncol. 2008;9(3):279-87.

19. Chey WD, et al. ACG clinical guideline: treatment of Helicobacter pylori infection. Am J Gastroenterol. 2017;112(2):212.

20. Miki K. Gastric cancer screening by combined assay for serum anti-Helicobacter pylori $\mathrm{IgG}$ antibody and serum pepsinogen levels_“ABC method”. Proc Jpn Acad Ser B. 2011;87(7):405-414.

Publisher's Note Springer Nature remains neutral with regard to jurisdictional claims in published maps and institutional affiliations. 